\title{
The Pre-PRIMA Survey Project: Turning the problem around
}

\author{
A. Richichi ${ }^{1}$, I. Percheron ${ }^{2}$, F. Delplancke ${ }^{1}$, and E.J. Bakker ${ }^{2}$ \\ 1 European Southern Observatory, Karl-Schwarzschildstr. 2, \\ D-85748 Garching b.M., Germany \\ 2 Leiden Observatory, University of Leiden, PO Box 9513, \\ NL-2300 RA Leiden, The Netherlands
}

\begin{abstract}
The mature life of the VLT Interferometer and its instrumentation will start with the availability of the PRIMA dual feed facility. Possible extensions of this latter might also be developed for use with second generation instrumentation, making it possible to combine and phase-reference several beams simultaneously. The introduction of the dual feed in interferometry will create a situation similar to that already experienced in Adaptive Optics, namely the fact that most scientific programs will be limited by the availability of a suitable reference star nearby. With sufficient time available ahead of us, we propose to turn the problem around and investigate the surroundings of a large number of suitably bright reference stars, to compile a list of interesting objects which could be studied by the VLTI and its dual feed facility. This would permit to obtain accurate astrometry and/or detailed imaging of objects such as brown dwarfs, (micro)gravitational lenses, extragalactic sources. We discuss the needs and strategies to investigate the surroundings of a number of bright stars, using available near-IR surveys, as well as novel dedicated observations.
\end{abstract}

\section{Limitations of interferometric facilities}

Interferometry works by measuring the visibility, or fringe contrast, of an astronomical source with a given baseline. It is estimated that the new generation interferometric facilities, based on 8-10 $\mathrm{m}$ telescopes such as the VLTI and the Keck, when aided by adaptive optics (AO), will reach a magnitude limit of 1214 in the near-infrared. This restriction is due to physical limitations a) on the characteristics of currently available detectors, and b) on the number of photons that can be recorded in a minimum integration time. The exposure time is generally set to a few milliseconds or tens of milliseconds, limited by the effect of atmospheric turbulence.

To extend significantly this sensitivity limit by integrating for longer times, it is necessary to stabilize the fringes, or in other words to "freeze" the atmospheric turbulence. The situation is analogous to that encountered for AO. Similarly to what has been done for this latter technique, in the field of interferometry there have been efforts [1] to use a nearby, bright reference star to stabilize and record the fringes. Then, if the science source is within the isopistonic patch [2] [3], integration times can be extended almost arbitrarily, apart from saturation effects. 


\section{The PRIMA Concept}

The VLTI will implement the method of using a bright reference stars for fringe tracking, in the PRIMA dual-feed facility. This optical device will permit to inject the light of two stars in the interferometer, which are separated by angular distances $<1^{\prime}$, and to monitor accurately the optical path differences of the (four) beams. The main characteristics of PRIMA are summarized in [4], [5], [6].

In practice, three main modes of operation will be available: 1) an astrometric mode, in which the angular distance between the two stars is measured with an accuracy as good as $10 \mu \mathrm{as}, 2)$ a faint source mode, in which the fringes are tracked on the bright reference star, and long integrations are performed on the scientific target, presumably much fainter. It is foreseen to reach magnitudes of about $\mathrm{K}=20$ in this way, 3) a phase-referenced imaging mode, where one of the two stars (usually the fringe tracking one) is used as a phase-reference (zero) for the science star fringes, allowing aperture synthesis imaging.

PRIMA will allow astrometry with an accuracy of $10 \mu$ as, over a total observation time of 30 minutes, if the stars are separated by 10 arcsec, with the Paranal median atmospheric turbulence ( 0.65 arcsec seeing).

The baseline vector is measured on calibration stars, as usual in interferometry. The OPD caused by the atmospheric turbulence is averaged toward zero by measuring the fringe positions and the internal metrology during a long enough period (typically $30 \mathrm{~min}$ ). The internal OPD is measured by a dedicated high accuracy laser metrology. Thus, the two parameters that can be measured are the angular star separation vector and the object phase, linked to its shape.

The phase-referenced imaging dynamic range will be limited by the number of baselines rather than by the visibility and phase measurement accuracies, which will be better than $1 \%$. However, the main PRIMA limitation will be the small size of the isopistonic patch $(\approx 20$ arcsec diameter in $\mathrm{K}$ band [3]) where proper astrometry and imaging can be performed.

\section{The Pre-PRIMA Survey Project}

For the purpose of this presentation, we concentrate ourselves on the modes (faint source mode and phase-referenced imaging mode) which require a reference star. It is clear that, with a limiting magnitude of about H or K 12 for the reference star, the sky coverage of this method of observation will not be complete. It follows that the use of PRIMA will be limited to a certain number of bright guide stars. On the other hand, the number of sources within the potential sensitivity limit is so high, that there will be no shortage of sources to observe around these bright stars. In particular, it is anticipated that the applications will cover all classes of astronomical sources, from young stars to faint extragalactic sources. To make the best use of the sensitivity limit in the relatively small sky areas available, it is sensible to investigate ahead in depth small areas of the sky around potential bright reference stars. For this, a project is being started between ESO and NEVEC, to carry out a survey around a number of 
such stars. The outline of the project is as follows:

a) Selection of reference objects. The selection will be done by investigating different lists of natural guide stars (NGS) for adaptive optics and other sky survey catalogues. We could also use the preliminary version of the VLTI catalogue [7], which is a compilation of published high angular resolution measurements (long baseline interferometry, aperture masking on big telescopes like Keck, WHT, SAO, and lunar occultations).

b) Pre-selection of scientific objects nearby a reference object. Areas around each reference object (see above) will be cross-correlated with catalogues at various wavelengths, from optical to IR (IRAS), X-rays (Rosat), and radio (NVSS, FIRST, WENSS), and with archive data (HST, ESO, La Palma).

c) Novel dedicated observations around the reference objects will be done including deep imaging of the fields around the reference objects using a large telescope with Adaptive Optics and with the option of using coronographic techniques. These observations will include: deep imaging (magnitudes and variability of objects, morphological selection); wide-range, low-medium resolution spectroscopy (to characterize extragalactic objects); high resolution spectroscopy (for special targets); monitoring of flux and radial velocity variations when required.

d) Following these observations the scientific targets will be characterised to derive: accurate relative position to the reference object; object type; magnitude; angular diameter, if available.

The project will provide a data base containing science targets around a set of few tens (which could be extended to more than 100) scientific targets with their associated bright reference NIGS (Natural Interferometric Guide Star). The project is expected to need about 4 man/years, and to be completed in 2005 .

\section{References}

1. Colavita, M.M. et al. 1999, ApJ, 510, 505.

2. S. Esposito, A. Riccardi, B. Femenia, 2000 A\&A, 353, L29

3. B. Femenia, M. Carbillet, S. Esposito, A. Riccardi, 2000, SPIE conference 4006, p. 1116

4. F. Delplancke, S. Leveque, A. Glindemann, P. Kervella, L. d' Arcio, 2000, SPIE conference 4006, 365

5. A. Glindemann, S. Leveque 2000, The VLT Opening Symposium, J. Bergeron and A. Renzini (eds), p. 468

6. Quirrenbach A. et al. Proc. SPIE Vol. 3350, p. 807

7. A. Richichi, I. Percheron, 2000, VLT-TRE-ESO-15000-2416 